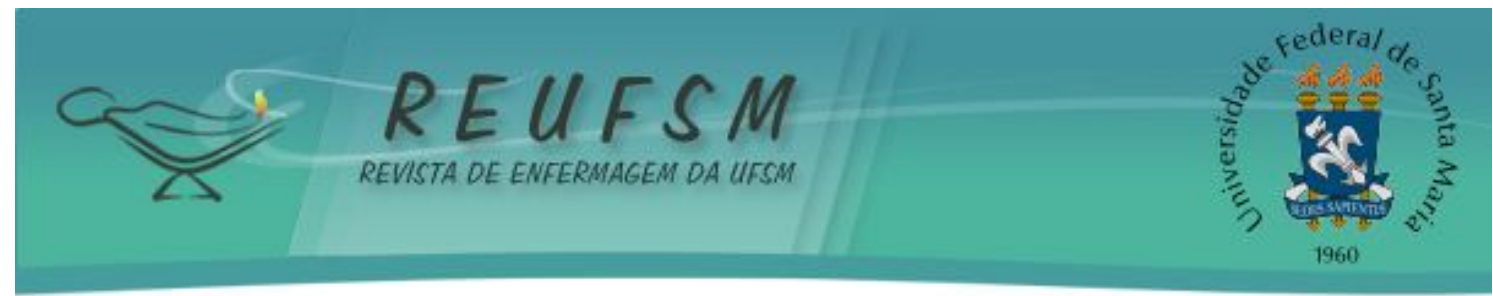

ARTIGO DE REVISÃO

\title{
UTILIZAÇÃO DO MODELO DEMANDA-CONTROLE DE KARASEK NA AMÉRICA LATINA: UMA PESQUISA BIBLIOGRÁFICA
}

\author{
USE OF KARASEK'S DEMAND-CONT ROL MODEL IN LATIN AMERICA: A BIBLIOGRAPHIC \\ RESEARCH
}

\section{UTILIZACIÓN DEL MODELO DEMANDA-CONT ROL DE KARASEK EN AMÉRICA LATINA: UNA REVISIÓN DE LA LITERAT URA}

\author{
Patrícia Bitencourt Toscani Greco ${ }^{1}$ \\ Tânia Solange Bosi de Souza Magnago ${ }^{2}$ \\ Andrea Prochnow ${ }^{3}$ \\ Carmem Lúcia Colomé Beck ${ }^{4}$ \\ Juliana Petri Tavares ${ }^{5}$
}

RESUMO: Objetivo: identificar as produções científicas que utilizaram o modelo demandacontrole na América Latina. Metodologia: pesquisa bibliográfica nas bases de dados LILACS, SCIELO E MEDLINE, realizada no período de junho a setembro de $2010 \mathrm{com}$ as palavraschave: "modelo demanda-controle", "Karasek", "job content questionnaire", "job stress scale" e os descritores: "estresse psicológico" e "saúde do trabalhador". Resultados: obteve-se 35 estudos (30 artigos e cinco teses). Quanto ao ano das publicações, destacamse os anos de 2009 e 2010 (40\%). O periódico com maior número de publicações foi a Revista de Saúde Pública do Brasil (20\%). Os estudos investigaram trabalhadores da área da saúde $(48,6 \%)$, docentes $(14,3 \%)$, outras áreas de trabalho e estudos técnicos. Conclusão: evidenciou-se a importância do modelo demanda-controle na investigação do ambiente psicossocial do trabalho, principalmente os efeitos do estresse ocupacional e suas repercussões sobre a saúde, em estudos com populações de diversas áreas.

Descritores: Enfermagem; Estresse psicológico; Saúde do trabalhador; Trabalho.

ABSTRACT: Objective: to identify the scientific productions that applied the demandcontrol model in Latin America. Methodology: it is a bibliographic research in the database of LILACS, SCIELO, and MEDLINE, conducted from J une to September 2010 with the keywords: "demand-control model", "karasek", "job content questionnaire", "job stress scale", and the descriptors: "psychological stress" and "occupational health". Results: 35 studies have been found (30 papers and five dissertations). The years of

\footnotetext{
${ }^{1}$ Enfermeira. Mestranda do Programa de Pós-Graduação em Enfermagem da UFSM. Bolsisita CAPES-REUNI. Membro do Grupo de Pesquisa Trabalho, Saúde, Educação, e Enfermagem do Departamento de Enfermagem da UFSM. Linha de pesquisa: Saúde do Trabalhador. Santa Maria, Rio Grande do Sul, Brasil. pbtoscani@hotmail.com

2 Enfermeira. Doutora em Enfermagem. Professor Adjunto do Departamento de Enfermagem da UFSM. Membro do Grupo de Pesquisa: Trabalho, Saúde, Educação e Enfermagem do Departamento de Enfermagem da UFSM Linha de Pesquisa Saúde do Trabalhador. Santa Maria, Rio Grande do Sul, Brasil.tmagnago@terra.com.br

3 Enfermeira. Mestranda do Programa de Pós-Graduação em Enfermagem da UFSM. Membro do Grupo de Pesquisa Trabalho, Saúde, Educação, e Enfermagem do Departamento de Enfermagem da UFSM. Linha de pesquisa: Saúde do Trabalhador. Santa Maria, Rio Grande do Sul, Brasil. andrea-zinha@hotmail.com

${ }^{4}$ Enfermeira. Doutora em Enfermagem. Professor Associado II do Departamento de Enfermagem da UFSM. Membro do Grupo de Pesquisa: Trabal ho, Saúde, Educação e Enfermagem da UFSM. Santa Maria, Rio Grande do Sul, Brasil. carmembeck@gmail.com

5 Enfermeira. Mestranda no Programa de Pós-Graduação em Enfermagem da Universidade Federal de Santa Maria (UFSM). Membro do Grupo de Pesquisa: Trabalho, Saúde, Educação e Enfermagem do Departamento de Enfermagem da UFSM- Linha de Pesquisa Saúde do Trabalhador. Santa Maria, Rio Grande do Sul, Brasil. jupetritavares@gmail.com
} 


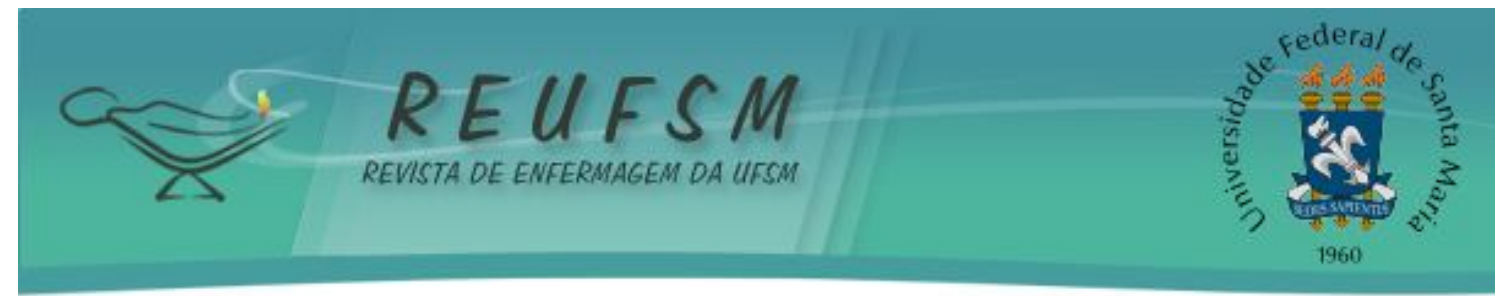

publication highlighted were 2009 and $2010(40 \%)$. The periodic with highest number of publications was Revista de Saúde Pública do Brasil (20\%). The studies researched workers in the health area $(48.6 \%)$, teachers $(14.3 \%)$, and other areas of work and technical studies. Conclusion: It was highlighted the importance of the demand-control model for psychosocial working environment investigations, mainly the effects of labor stress and its repercussions on health, in studies with populations of many areas.

Descriptors: Nursing; Stress, Psychological; Occupational health; Work.

RESUMEN: Objetivo: identificar los estudios científicos que han utilizado el modelo demanda-control en América Latina. Metodología: búsqueda bibliográfica en las bases de datos LILACS y SCIELO MEDLINE, realizada de junio a septiembre de 2010, con Ias palabras clave: "modelo de demanda-controle", "Karasek", "job content questionnaire", "job stress scale", y las palabras: "estresse psicológico" y "saúde do trabalhador". Resultados: se obtuvieron 35 estudios (30 artículos y cinco tesis). En cuanto al año de la publicación, se destacan los años 2009 y 2010 (el 40\%). El periódico con el mayor número de publicaciones fue el Diario de Salud Pública de Brasil (el 20\%). Los estudios investigaron trabajadores de la salud (el 48,6\%), profesores (el 14,3\%), otras áreas de trabajo y estudios técnicos. Conclusión: el estudio mostró la importancia del modelo demandacontrol en la investigación del entorno psicosocial de trabajo, especialmente los efectos del estrés laboral y sus consecuencias sobre la salud en estudios de población de diversas áreas.

Descriptores: Enfermería; Estrés psicológico; Salud Iaboral; Trabajo.

\section{INTRODUÇÃO}

Na década de 70 , as pesquisas sobre as repercussões do trabalho na saúde dos trabalhadores, são influenciadas pelas observações e estudos experimentais de Hans Selye. Apesar dos avanços conceituais, os estudos eram unidimensionais baseados principalmente nas demandas das tarefas ou se detinham nas demandas versus capacidades do indivíduo no enfrentamento destas. 0 controle do trabalhador sobre o processo de trabalho não fazia parte da análise dos processos de produção de desgaste laboral. ${ }^{1}$

Esse enfoque limitado dos modelos unidimensionais colocou em discussão a necessidade de estarem presentes, concomitantemente, controle e demanda na abordagem da experiência psicossocial no trabalho e suas repercussões sobre a saúde dos trabalhadores ${ }^{2}$. Nessa perspectiva, no final da década de 70, Robert Karasek propôs um novo modelo de investigação chamado Modelo Demanda-Controle (Demand-Control Model ou J ob Strain). Este modelo bi-dimensional relaciona dois aspectos psicossociais no ambiente de trabalho (demandas psicológicas e controle do trabalhador sobre o trabalho) ao risco de adoecimento. ${ }^{3}$

A demanda psicológica está relacionada às exigências psicológicas que o trabalhador enfrenta para concretizar suas atividades (pressão do tempo, nível de concentração, interrupção das tarefas e a necessidade de aguardar pelas atividades de outros trabalhadores). 0 controle do trabalhador sobre 0 trabalho relaciona-se a dois aspectos: ao uso de habilidades (o grau que o trabalho envolve a aprendizagem, repetitividade e criatividade) e a autoridade decisória (habilidade para a tomada de decisões no trabalho e influências na política gerencial). ${ }^{3}$

A avaliação do ambiente psicossocial do trabalho é realizada a partir da combinação de níveis altos e baixos dessas duas dimensões, configurando-se em quatro situações específicas de trabalho que sugerem riscos diferenciados à saúde: alta exigência (alta demanda e baixo controle) são as reações mais adversas de desgaste psicológico; trabalho ativo (alta demanda psicológica e alto controle) permite ao trabalhador ter uma 


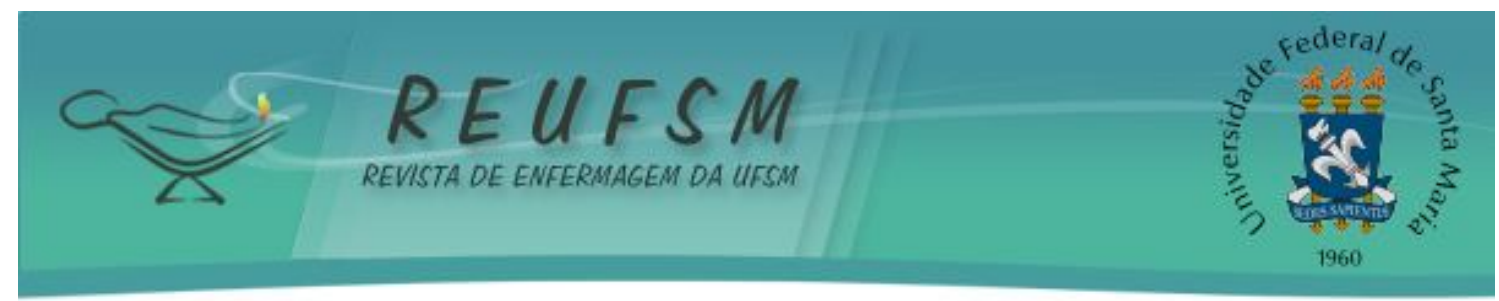

ampla possibilidade de decisão sobre como e quando desenvolver suas tarefas, bem como usar sua potencialidade intelectual com esta finalidade; trabalho passivo (baixa demanda psicológica e baixo controle) produz uma atrofia gradual de aprendizagem de habilidades e baixa exigência (combina baixa demanda e alto controle) que se configura num estado altamente confortável e ideal de trabalho. ${ }^{3}$

De acordo com os pressupostos deste modelo $0^{3} 0$ estresse ocupacional é resultante da interação entre altas demandas psicológicas, menor controle no processo de produção do trabalho e menor suporte social recebido de colegas e chefes. Essa condição pode acarretar consequências nocivas à saúde física ou mental do trabalhador, ou seja, os trabalhadores que se defrontam com altas demandas ou pressões psicológicas no trabalho combinadas com um baixo controle ou baixo poder de decisão correm um grave risco de adoecimento em decorrência do desgaste psicológico. ${ }^{3}$

O Modelo Demanda-Controle procura explicar as modificações que acontecem com os indivíduos submetidos à tensão no trabalho, tanto em nível fisiológico quanto psicológico. Ele tem sido apontado como possibilidade de estrutura integradora para estudo dos diversos elementos do ambiente de trabalho, nas suas inter-relações com a saúde dos trabalhadores. ${ }^{4.5}$

Estudos realizados, principalmente, em países da Europa, da Ásia e nos Estados Unidos da América têm evidenciado associação positiva entre aspectos psicossociais do trabalho (alta demanda psicológica e baixo controle) e diferentes desfechos, como, por exemplo: problemas psiquiátricos menores ${ }^{6}$, doenças do sistema digestivo ${ }^{3}$, desordens musculoesqueléticas ${ }^{7-9}$, doenças cardiovasculares ${ }^{10-11}$, entre outros.

Na última década houve uma expansão do uso do Modelo Demanda-Controle em outros países, dentre eles os da América Latina. Portanto, este estudo se propõe a fazer um levantamento dos estudos produzidos e publicados, nesta parte do continente Americano, no intuito de encontrar lacunas para novas pesquisas.

Assim, este estudo tem como objetivo identificar as produções científicas que utilizaram o Modelo Demanda-Controle na América Latina no período de 1979 a 2010.

\section{MÉTODO}

Trata-se de uma pesquisa bibliográfica acerca da utilização do Modelo DemandaControle nas produções científicas na área da saúde do trabalhador, na América Latina no período de 1979 (ano de criação do modelo) a 2010. Incluíram-se como critérios: publicações latino-americanas existentes nas bases de dados Scientific Electronic Library Online (SCIELO), Literatura Latino-Americana em Ciências da Saúde (LILACS) e Medical Literature Analysis and Retrieval System Online (MEDLINE) que utilizassem, como referencial teórico ou metodológico o Modelo Demanda-Controle de Karasek, ou pesquisa bibliográfica sobre o mesmo.

O levantamento de dados foi realizado no período de julho a setembro de 2010, com as seguintes palavras-chave: "Modelo Demanda-controle", "Karasek", "J ob Content Questionnaire" (J CQ), "J ob Stress Scale" (J SS). Foram utilizadas palavras-chave por não existir descritor que caracterize esse modelo. Após a observação dos descritores utilizados nos artigos selecionados, verificou-se o uso frequente de dois descritores: "estresse psicológico" e "saúde do trabalhador". Estes também foram utilizados em uma nova busca nas bases de dados supracitadas.

$\mathrm{Na}$ sel eção inicial, ao serem utilizadas as palavras-chave, uma a uma, de forma isolada, obteve-se 1.236 estudos. Quando utilizados os descritores "estresse psicológico" and "saúde do trabalhador" numa busca avançada, foram encontrados mais 801, totalizando 2.037 estudos.

Para a seleção dos artigos, fez-se a leitura dos títulos e resumos, selecionando-se aqueles com enfoque na temática em foco. Do total de artigos, foram excluídos 2.002 por não utilizarem o Modelo Demanda-Controle (MDC) como referencial na investigação ou pela repetição dos estudos nas diferentes bases de dados. Com isso, foram incluídos 35 estudos, para análise. 


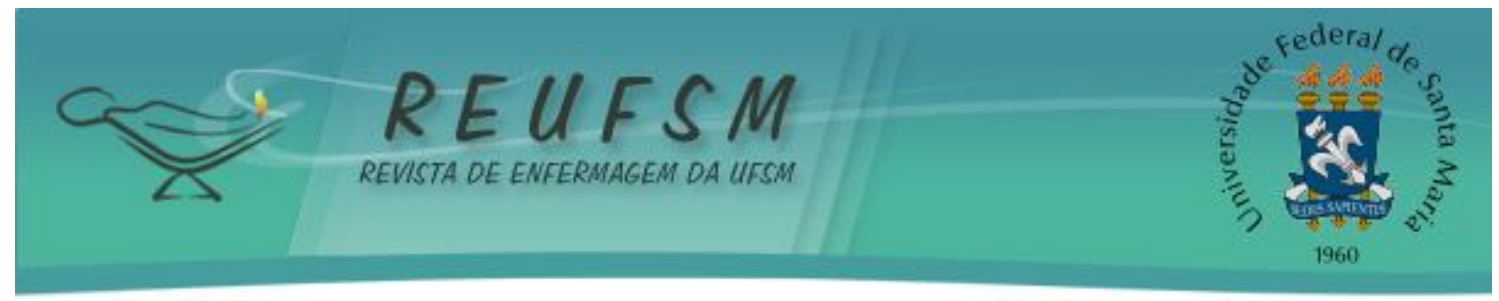

Após, prosseguiu-se a busca dos artigos completos e das teses. Com a posse dos textos na íntegra, realizou-se a leitura crítica dos manuscritos, extraindo-se, em formulário próprio, informações específicas referentes à nacionalidade, ao periódico e ano de publicação, à titulação dos autores, aos cenários das pesquisas, aos suj eitos investigados e aos resultados encontrados.

\section{RESULTADOS E DISCUSSÃO}

Dos 35 estudos selecionados, $85,7 \%(\mathrm{~N}=30)$ referem-se a artigos científicos publicados em periódicos e $14,3 \%(\mathrm{~N}=5)$ a teses produzidas nos Programas de PósGraduação do Brasil. Dos artigos, 13,3\% ( $N=4)$ encontravam-se publicados em periódicos mexicanos e os demais em periódicos brasileiros (86,7\%).

Quanto ao ano de publicação nos periódicos, o ano de 1997 marca a primeira publicação nas bases de dados consultadas. Refere-se a um estudo que avalia a associação entre condições de trabalho materno e baixo peso ao nascer na Cidade do México. ${ }^{12}$ No Brasil, o estudo pioneiro é uma tese defendida em 1999, que avaliou a associação entre as condições de trabalho e distúrbios psíquicos menores em trabal hadoras de enfermagem, na cidade de Salvador - Bahia. ${ }^{4}$

No período de 2000 a 2002 não foram encontradas publicações sobre a temática. Em 2003 e 2004, localizaram-se cinco artigos publicados (14,3\%) e uma tese $(2,85 \%)$ em 2004 . Em 2005 e 2006, foram publicados cinco artigos (14,3\%) e, nos anos de 2007 e 2008, localizaram-se seis artigos (17,14\%) e duas teses (5,71\%). Por fim, os anos de 2009 e 2010, representaram 0 maior número de publicações na forma de artigo $(37,14 \% \mathrm{~N}=13)$ e uma tese $(2,85 \%)$.

Em relação à fonte de publicação, os artigos foram encontrados em 16 periódicos diferentes, dos quais cinco del es são específicos da enfermagem. A Tabela 1 apresenta 0 número de artigos publicados por periódico durante o período avaliado.

Tabela 1 - Distribuição dos artigos que utilizaram o Modelo Demanda-Controle e publicados na América-Latina, segundo o periódico de publicação, 1997 a 2010. $(N=30)$

\begin{tabular}{lc}
\hline Revistas & № de artigos publicados \\
\hline Revista de Saúde Pública & 6 \\
Revista Salud Pública do México & 4 \\
Caderno de Saúde Pública & 3 \\
Ciência e Saúde Coletiva & 3 \\
Revista Texto \& Contexto Enfermagem & 2 \\
Revista de Enfermagem da Universidade Estadual do Rio de janeiro & 2 \\
Revista Brasileira de Terapia Intensiva & 1 \\
Revista Fisioterapia Brasil & 1 \\
Revista da Associação Médica Brasileira & 1 \\
Nursing & 1 \\
Revista Gaúcha de Enfermagem & 1 \\
Interamerican J ournal of Psychology & 1 \\
Caderno de Psicologia Social e Trabalho & 1 \\
Revista Brasileira de Psiquiatria & 1 \\
Educação e Sociedade & 1 \\
Revista Latino Americana de Enfermagem & 1 \\
\hline Total & 30 \\
\hline
\end{tabular}




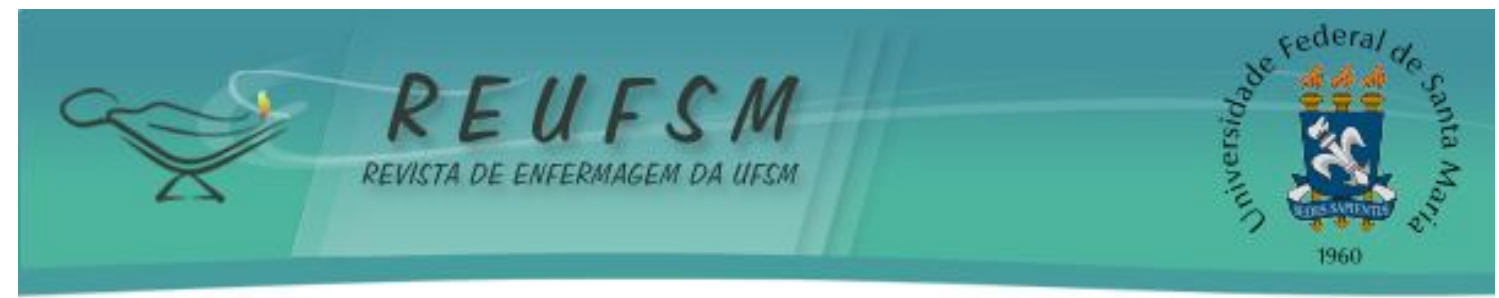

Tendo em vista que os estudos estão inseridos no contexto da saúde do trabalhador e, muitos deles, caracterizados como estudos epidemiológicos, compreende-se o maior número de publicações em periódicos de saúde pública.

As teses representam $14,3 \%(\mathrm{~N}=5)$ dos estudos localizados. A primeira tese utilizando o MDC foi defendida em 1999, na Universidade Federal da Bahia. Aborda questões do trabalho e distúrbios psíquicos em mulheres trabalhadoras de enfermagem, evidenciando associação positiva entre alta exigência no trabalho e distúrbios psíquicos menores ${ }^{4}$.

Em 2004, na Escola Nacional de Saúde Pública do Rio de Janeiro, a segunda tese, proveniente do Estudo Pró-saúde, não evidenciou associação entre alta exigência e hipertensão arterial em mulheres. ${ }^{5}$ Em 2007, outra tese relaciona estresse ocupacional e acidentes de Trabalho na Universidade do Estado de Rio de J aneiro. ${ }^{13}$

Em 2008, na Escola de Enfermagem Anna Nery, da Universidade Federal do Rio de Janeiro uma tese relacionou os aspectos psicossociais do trabalho e distúrbios musculoesqueléticos em trabalhadores de enfermagem, evidenciando associação positiva entre alta exigência no trabalho e dor nos ombros, na coluna torácica e nos tornozelos. ${ }^{14}$ No ano de 2009, na Universidade Estadual do Rio de Janeiro, discutiu-se em uma tese os aspectos psicossociais do impedimento laboral por motivos de saúde em trabalhadores de cozinhas industriais, destacando-se associação entre alta demanda psicológica e acidentes no trabalho. ${ }^{15}$

Em decorrência das características dos estudos os dados foram divididos em dois eixos temáticos: Estudos epidemiológicos e Estudos técnicos. Neste último, foram considerados os estudos que abordaram: as contribuições do MDC; a tradução e validação da versão resumida da escala do MDC.

\section{Estudos epidemiológicos}

Os estudos epidemiológicos foram agrupados em três categorias: trabalhadores da área da saúde, docentes e outras áreas de trabalho.

Trabalhadores da área da saúde

Nesta categoria estão alocados $48,6 \%(\mathrm{~N}=17)$ dos estudos, sendo que se destacam como população os profissionais de enfermagem com $40 \%(\mathrm{~N}=14)$, os médicos com $2,8 \%$ $(N=1)$ e $5,7 \%(N=2)$ dos estudos agregam médicos e enfermeiros. Observa-se que dos estudos realizados com a enfermagem, todos investigam profissionais que atuam no ambiente hospitalar. Quando relacionado aos médicos, dois artigos referem-se a profissionais de unidades de terapia intensiva ${ }^{16-17}$, e um refere-se a uma amostra aleatória de médicos em Salvador/Bahia ${ }^{18}$.

Os principais desfechos avaliados nos trabalhadores da saúde foram: qualidade de $v^{v i d a}{ }^{16}$, distúrbios musculoesqueléticos ${ }^{19}$, pressão arterial e sintomas cardiovasculares ${ }^{20}$, síndrome da estafa profissional (burnout) ${ }^{17}$, distúrbios psíquicos menores ${ }^{1,18,21}$ e acidentes de trabalho com material biológico 22 . Destaca-se que alguns estudos evidenciaram associação positiva entre alta exigência no trabalho e os desfechos avaliados ${ }^{1,17-21}$, ou seja, os trabalhadores expostos à alta demanda psicológica e a baixo controle sobre o trabalho apresentam maiores chances de adoecerem, corroborando a hipótese do MDC.

Com relação ao trabalho da enfermagem é importante considerar algumas características inerentes a profissão: ser uma profissão interdependente, intercomplementar, com uma autonomia relativa no que se refere aos demais trabalhadores da área de saúde e aos pacientes. Ainda, a enfermagem é considerada como 


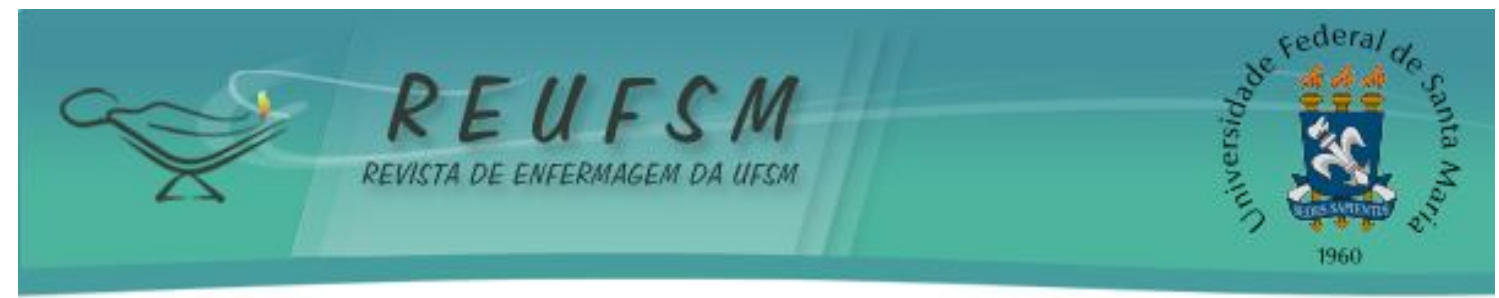

uma profissão em que os trabalhadores estão suscetíveis ao estresse por trabalhar com pacientes com doenças críticas, com situações limítrofes da vida e de morte. Tais características aliadas ao trabalho em turnos compõe um cenário desgastante que a torna suscetível ao adoecimento. ${ }^{23-24}$

No que tange à atividade médica, os estudos apontam a medicina intensivista como uma especialidade estressante, por vários motivos: conflito com o objetivo da cura, maior exposição à morte, exigência de conhecimento e habilidades técnicas qualificados, atenção, raciocínio rápido, controle emocional, bem como a necessidade constante de atualização devido ao crescente desenvolvimento tecnológico. ${ }^{17-18}$

Essas características (proximidade com a morte, necessidade de controle, qualificação, entre outras) tornam a atividade dos profissionais da saúde, que atuam no cenário hospitalar, estressante, pois é nesse ambiente laboral que se estabelecem as demandas psicológicas e nele o profissional experimenta variados graus de controle sobre as atividades que executa. ${ }^{21}$

\section{Docentes}

No que se refere à categoria dos docentes, foram localizados $14,3 \%(N=5)$ estudos. A maioria deles foi realizado na rede municipal de ensino e estabeleceram relação com os seguintes desfechos: distúrbios psíquicos menores ${ }^{25-}{ }^{26}$, qualidade de vida ${ }^{27}$ e exaustão ${ }^{28}$. Nesses estudos foi evidenciada associação positiva entre 0 desfecho avaliado e a alta exigência no trabalho (alta demanda psicológica e baixo controle), quando comparados aos docentes classificados em situação de baixa exigência no trabalho (baixa demanda psicológica e alto controle).

Cabe destacar que apesar do trabalho docente propiciar certa liberdade e criatividade (no sentido pedagógico e em sala de aula), permitindo um maior controle sobre algumas questões inerentes ao trabalho, ao mesmo tempo, os docentes estão submetidos a atividades extraclasse, extensa jornada de trabalho, cumprimento de tarefas com prazo curto de tempo e múltiplos empregos. O somatório dessas características pode favorecer aos efeitos negativos sobre a saúde docente. ${ }^{25}$

Nesta revisão, os acometimentos mais relatados foram: doenças cardiovasculares, labirintite, faringite, fadiga, neuroses, insônia e tensão nervosa. Considera-se que a desvalorização profissional e a baixa auto-estima são fatores que favorecem para que os resultados encontrados nas pesquisas com docentes sejam de exaustão, alta prevalência de distúrbios psíquicos menores, desgaste psicológico, cansaço mental e nervosismo. ${ }^{28-29}$

Destaca-se que quando comparados a outros trabalhadores pesquisados na área da saúde ocupacional, os professores possuem uma relativa carência de estudos. ${ }^{28}$

\section{Outras categorias profissionais}

No relatório da Europen Agency for Safety and Health at Work, "...estresse pode ser experienciado como o resultado de exposições a uma ampla variedade de demandas de trabalho e, por sua vez, contribui igualmente para uma ampla variedade de resultados sobre a saúde dos trabalhadores". ${ }^{30: 50}$ Nesse sentido, cabe destacar que o estresse permeia todas as atividades laborais e repercute na saúde dos trabalhadores, independente de área de atuação.

Do total de estudos revisados, $28,6 \%(\mathrm{~N}=10)$ avaliaram populações variadas como: funcionários técnico administrativo, adolescentes e mulheres trabalhadoras, servidores de um tribunal, trabalhadores de uma empresa de transporte coletivo, de cozinhas industriais, de linhas de produção e trabalhadores de empresas mexicanas (sendo que o 


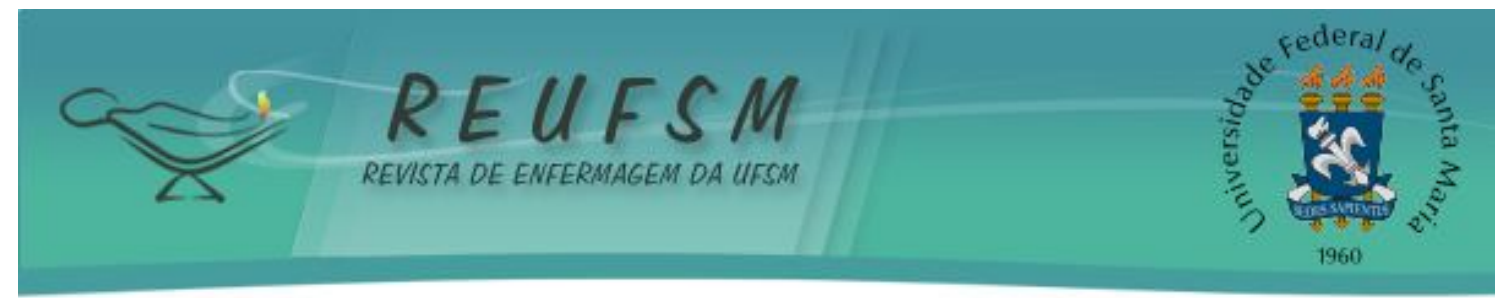

último não especifica a área de atuação dos trabalhadores). As investigações tiveram por finalidade verificar a existência de associação entre alta exigência no trabalho e: condições de vida e estado de saúde, Índice de Capacidade para o Trabalho (ICT), impedimento laboral por motivos de saúde e sintomas de estresse.

Várias situações no trabalho são consideradas de risco para desenvolver o estresse e danos à saúde do trabalhador. ${ }^{31}$ Foram destacados como agentes estressantes: as demandas acima das condições efetivas de produção ou prestação de serviços de qualidade, 0 insuficiente reconhecimento profissional, a pequena participação nas decisões organizativas, as longas jornadas de trabalho, a exposição constante ao risco e periculosidade, a pressão do tempo e atuações de urgência, os problemas de comunicação, a competitividade e 0 excesso de burocracia. ${ }^{32}$

Faz-se necessário e urgente a adoção de medidas para o reconhecimento, a prevenção e controle do estresse relacionado ao trabalho. ${ }^{32}$ Aliás, essa tem se evidenciado como uma preocupação internacional. ${ }^{32-33}$

\section{Estudos técnicos}

Nesta categoria, encontraram-se dois estudos, sendo que um deles apresenta as contribuições do MDC e o outro a versão resumida da J ob Stress Scale (J SS).

0 estudo relacionado às contribuições do MDC relata a possibilidade de articulação de esforços e integração de pesquisadores de diferentes países, no intuito de estudar os efeitos do estresse ocupacional sobre a saúde dos trabalhadores, abordando suas diversas repercussões: doenças cardiovasculares, distúrbios psíquicos, doenças osteomusculares e outros. Esse estudo apresenta os pressupostos teóricos e metodológicos do modelo, discute a capacidade do MDC nas diferentes situações de trabalho no contexto brasileiro, e descreve os achados sobre as repercussões na saúde mental em estudos que utilizaram o MDC. Apresenta as prevalências de distúrbios psíquicos menores em grupos distintos de acordo com as situações laborais preconizadas no MDC: baixa exigência, trabalho passivo, trabalho ativo e alta exigência. ${ }^{1}$

0 estudo sobre a versão resumida da J ob Stress Scale descreve o processo de tradução e adaptação dessa versão para o português, contribuindo com o desenvolvimento de pesquisas relacionadas ao estresse no ambiente de trabalho, no país. ${ }^{5}$ A JSS foi elaborada por Törres Theörell, em 1988, na Suécia, a partir da versão original do J ob Content Questionnaire (JCQ, 49 questões). A versão resumida possui 17 questões, sendo que cinco avaliam demanda psicológica, seis avaliam controle sobre o trabalho e seis investigam 0 apoio social. ${ }^{5}$

As relações entre trabalho, estresse e seus reflexos na saúde dos trabalhadores vem sendo abordada em estudos com abordagens metodológicas diferenciadas. O MDC é uma alternativa importante para proporcionar uma base integradora na diversidade metodológica, teórica e conceitual, principalmente referentes à intervenção nos ambientes laborais. Portanto, torna-se importante na produção do conhecimento envolvendo estresse, saúde e trabalho em contextos ocupacionais diferentes. ${ }^{1}$

Neste sentido, o referencial teórico-metodológico do MDC permite a avaliação das características do trabalho e seus efeitos no trabalhador, possibilitando o planejamento de ações voltadas à organização do trabalho com o intuito de promover a saúde do trabalhador. 


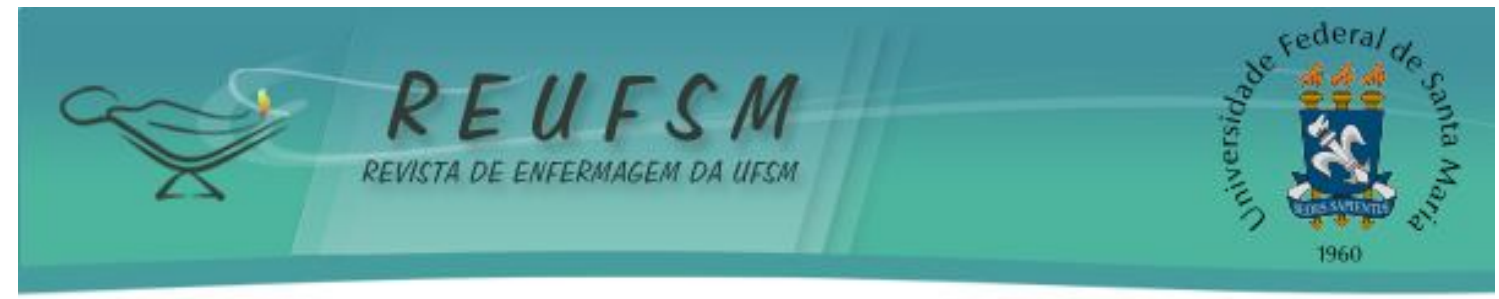

\section{CONSIDERAÇÕES FINAIS}

A análise das produções revisadas nesta pesquisa bibliográfica evidencia a importância do MDC na investigação do ambiente psicossocial do trabalho e os efeitos do estresse ocupacional sobre a saúde, abordando suas diversas repercussões. Ainda, é importante ressaltar a possibilidade de integração do MDC a outros instrumentos que avaliam, por exemplo, a saúde mental, a qualidade de vida, a síndrome da estafa profissional, acidentes de trabalho e absenteísmo, entre outros, contribuindo sobremaneira no campo da saúde do trabalhador.

A partir desta pesquisa, percebem-se investimentos em estudos com populações que apresentam riscos de adoecimento, de acordo com as condições impostas pelo trabalho, como trabalhadores da área da saúde e docentes. Como contribuição para novos estudos, este estudo evidencia a necessidade de reflexões sobre a relação entre o adoecimento e os níveis de demanda psicológica e de controle do trabalhador sobre o trabalho em outras profissões. Sugerem-se investigações, com policiais, bombeiros, agentes penitenciários, monitores de instituições para adolescentes, agentes sócio educadores, motoristas, dentre outras profissões estressantes ainda não investigadas com o referencial discutido neste artigo.

Como limitação do estudo, considerou-se a escassez de descritores específicos que caracterizem a temática, o que dificultou a busca dos estudos.

\section{REFERÊNCIAS}

1. Araújo TM, Graça CC, Araújo E. Estresse ocupacional e saúde: contribuições do Modelo Demanda- Controle. Ciênc Saúde Coletiva. 2003; 8(4): 991-1003.

2. Karasek RA. J ob Demand, job decision latitude, and mental strain: implications for job redesign. Adm Sci Q. 1979; 24:285-308.

3. Karasek RA, Theörell T. Healthy work-stress, productivity, and the reconstruction of working life. New York: Basic Books; 1990.

4. Araúj 0, TM. Trabalho e distúrbios psíquicos em mulheres trabalhadoras de enfermagem [tese de doutorado]. Salvador ; Universidade Federal da Bahia. Instituto de Saúde Coletiva ; 1999. 211 p.

5. Alves MGM, Chor D, Faerstein E, Lopes CS, Werneck GL. Versão resumida da "job stress scale": adaptação para o português. Rev Saúde Pública. 2004; 38(2): 164-71.

6. Harding TW, Arango MV, Baltazar J, Climent CE, Ibrahim HH, Ladrido-Ignacio L, et al. Mental disorders in primary health care: a study of their frequency and diagnosis in four developing countries. Psychol Med. 1980; 10:231-41.

7. J osephson M, Lagerström M, Hagberg M, Hjelm EW. Musculoskeletal Symptons and job strain among nursing personnel: a study over a three year period. Ocup Environ Med. 1997; 54(9): 681-5.

8. Bongers PM, Winter CR, Kompier M, Hilderbrandt V. Psychosocial factors at work and musculoskeletal disease. Scand J Work Environ Health. 1993; 19(5): 297-312.

9. Bongers PM, Kremer AM, Laak JT. Are psychosocial factors, risk factors for symptoms and signs of the shoulder, elbow, or hand/wrist?: A review of the epidemiological literature. Am J Ind Medicine. 2002; 41(5): 315-42. 


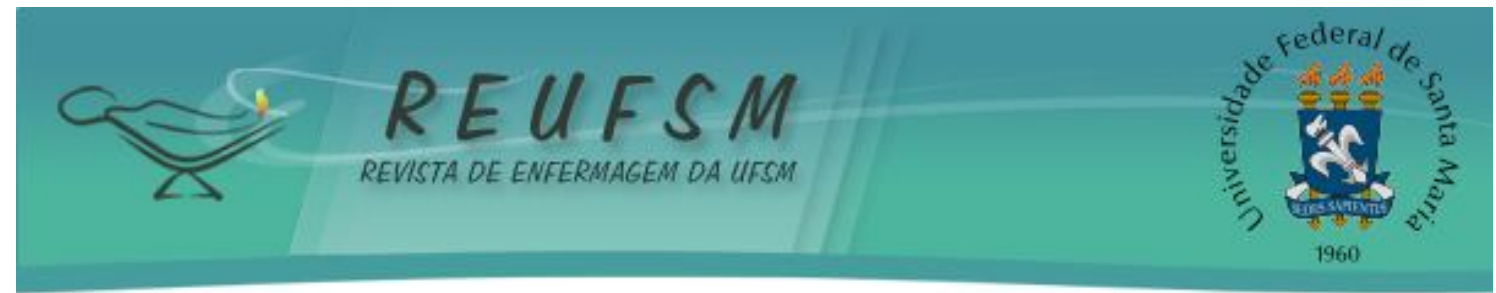

10. Theörell T, Karasek RA. Current issues relating to psychosocial job strain and cardiovascular diseasse research. J Occup Health Psychol. 1996; 1:9-26.

11. Schnall P, Landsbergis $P$, Baker D. J ob strain and cardiovascular disease. Annual Review of Public Health. 1994; 15:381-411.

12. Mireles BC, Carrillo CIS, Harlow SD, Urquiza RMN. Condiciones de trabajo materno y bajo peso al nacer en la Ciudad de México. Salud pública Méx. 1997; 39(1): 2-10.

13- Brito AS. Estresse e acidentes no trabalho: Estudo Pró-Saúde [tese de doutorado]. Rio de J aneiro: Universidade do Estado do Rio de J aneiro. Instituto de Medicina Social, 2007. $156 \mathrm{p}$.

14. Magnago TSBS. Aspectos psicossociais do trabalho e distúrbios musculoesqueléticos em trabalhadores de enfermagem [tese de doutorado]. Rio de Janeiro: Universidade Federal do Rio de J aneiro. Escola de Enfermagem Anna Nery, 2008. 201p.

15. Aguiar OB. Aspectos psicossociais do impedimento laboral por motivos de saúde em trabalhadores de cozinhas industriais [tese de doutorado]. Rio de J aneiro: Universidade do Estado do Rio de J aneiro. Instituto de Medicina Social, 2009. 206 p.

16. Fogaça MC, Carvalho WB, Nogueira PCK, Martins LAN. Estresse ocupacional e suas repercussões na qualidade de vida de médicos e enfermeiros intensivistas pediátricos e neonatais. Rev Bras Ter Intensiva. 2009; 21(3): 299-305.

17. Tironi MOS, Sobrinho CLM, Barros DS, Reis EJ FB, Filho ESM, Almeida A et al. Trabalho e síndrome da estafa profissional (Síndrome de Burnout) em médicos intensivistas de Salvador. Rev Assoc Med Bras. 2009; 55(6): 656-662.

18. Sobrinho CLN, Carvalho FM, Bonfim TAS, Cirino CAS, Ferreira IS . Condições de trabalho e saúde mental dos médicos de Salvador, Bahia, Brasil. Cad Saúde Pública. 2006; 22(1): 131-140.

19. Magnago TSBS, Lisboa MTL, Griep RH, Kirchhof ALC, Guido LA. Aspectos psicossocias do trabalho e distúrbios musculoesqueléticos em trabalhadores de enfermagem. Rev LatinoAm Enfermagem. 2010; 18(3): 140-47.

20.Garcia AJ. Factores psicosociales laborales relacionados con la tensión arterial y síntomas cardiovasculares en personal de enfermería en México. Salud pública México. 2007; 49(2): 109-117.

21. Kirchhof ALC, Magnago TSBS, Camponogara S, Griep RH, Tavares JP, Prestes FC et al. Condições de trabalho e características sócio-demográficas relacionadas à presença de distúrbios psíquicos menores em trabalhadores de enfermagem. Texto Contexto Enfermagem. 2009; 18(2): 215-223.

22. Dalarosa MG, Lautert L. Acidente com material biológico no trabalhador de enfermagem em um hospital de ensino: estudo caso-controle. Rev Gaúcha de Enfermagem. 2009; 30(1): 19-26.

23. Stacciarini J MR, Tróccoli BT. O estresse na atividade ocupacional do enfermeiro. Rev Latino-Am Enfermagem. 2001; 9(2): 17-25.

24. Pinho PS, Araújo TM. Trabalho de enfermagem em uma unidade de emergência hospitalar e transtornos mentais. Rev Enfermagem UERJ . 2007; 15(3): 329-336. 


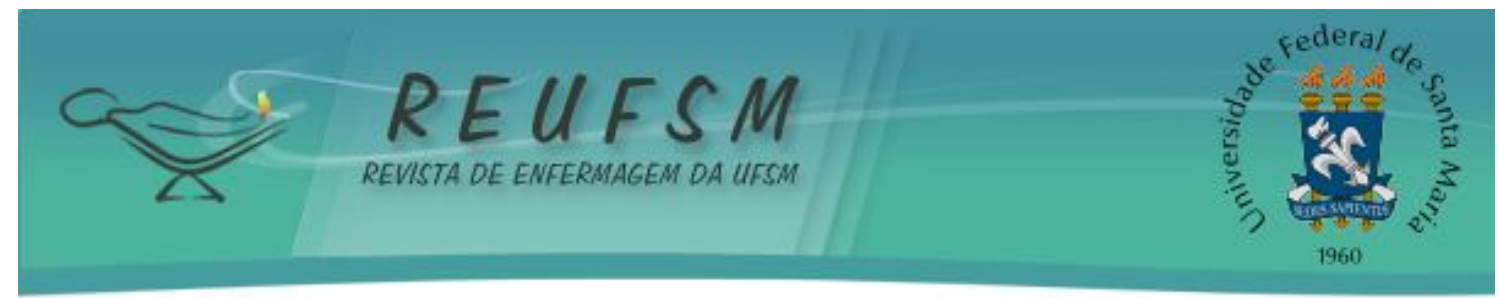

25. Reis EJ FB, Carvalho FM, Araújo TM, Porto LA, Neto AMS. Trabalho e distúrbios psíquicos em professores da rede municipal de Vitória da Conquista, Bahia, Brasil. Cad Saúde Pública. 2005; 21(5): 1480-1490.

26. Porto LA, Carvalho FM, Oliveira NF, Neto AMS, Araújo TM, Reis EJ FB et al. Associação entre distúrbios psíquicos e aspectos psicossociais do trabalho de professores. Rev Saúde Pública. 2006; 40(5): 818-826.

27. Fernandes MH, Rocha VM. Impacto dos aspectos psicossociais do trabalho na qualidade de vida dos professores. Rev Bras Psiquiatr. 2009 ; 31(1): 15-20.

28. Reis EJ FB, Araújo TM, Carvalho FM, Barbalho L, Silva MO. Docência e exaustão emocional. Educ Soc. 2006; 27(94): 229-253.

29. Delcor NS, Araújo TM, Reis EJ FB, Porto LA, Carvalho FM, Silva MO et al . Condições de trabalho e saúde dos professores da rede particular de ensino de Vitória da Conquista, Bahia, Brasil. Cad Saúde Pública. 2004; 20(1): 187-196.

30. Aptel MO, Cnockaert JM. Stress and work related musculoskeletal disorders of the upper extremities. France: Tutb Newletter. 2002, 19(20): 50-56.

31. Cooper G L. Handbook of stress, medicine and health. Boca Raton: CRC Press ; 1996. p. 3-25.

32. Zanelli J C, Calzaretta AV, Garcia AJ , Lipp MEN, Chambel MJ . Estresse nas Organizações de trabalho-compreensão e Intervenções baseadas em evidências. Porto Alegre: Artmed ; 2010.

33. Richardson KM, Rothstein HR. Effects of occupational stress management intervention programs: a meta-analysis. J Occup Health Psychol. 2008; 13(1): 69-93.

Data de recebimento: 16/02/2011

Data de aceite: 06/04/2011

Contato com autora responsável: Patrícia Bitencourt Toscani Greco.

Endereço: Rua Duque de Caxias, 517 bloco D1 ap.102. CEP: 97700-000. Santiago/ RS.

E-mail: pbtoscani@hotmail.com 